\title{
Maxillofacial Radiology 194
}

\author{
SADJ October 2021, Vol. 76 No. 9 p567
}

Jaco Walters ${ }^{1}$

CASE

A 19-year-old female presented with a firm tender swelling in the posterior left mandible. Teeth present in the quadrant tested vital with no discernible carious lesions. A pantomograph (Figure 1) revealed a mixed radiopaque mass that appears to be associated with the 36 roots. What would your provisional diagnosis be?
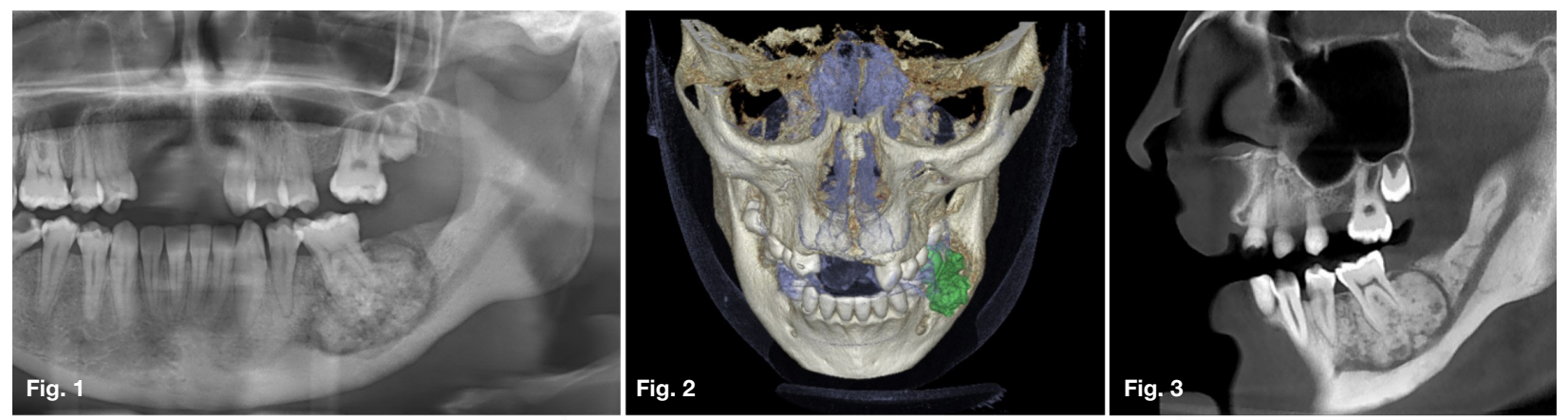
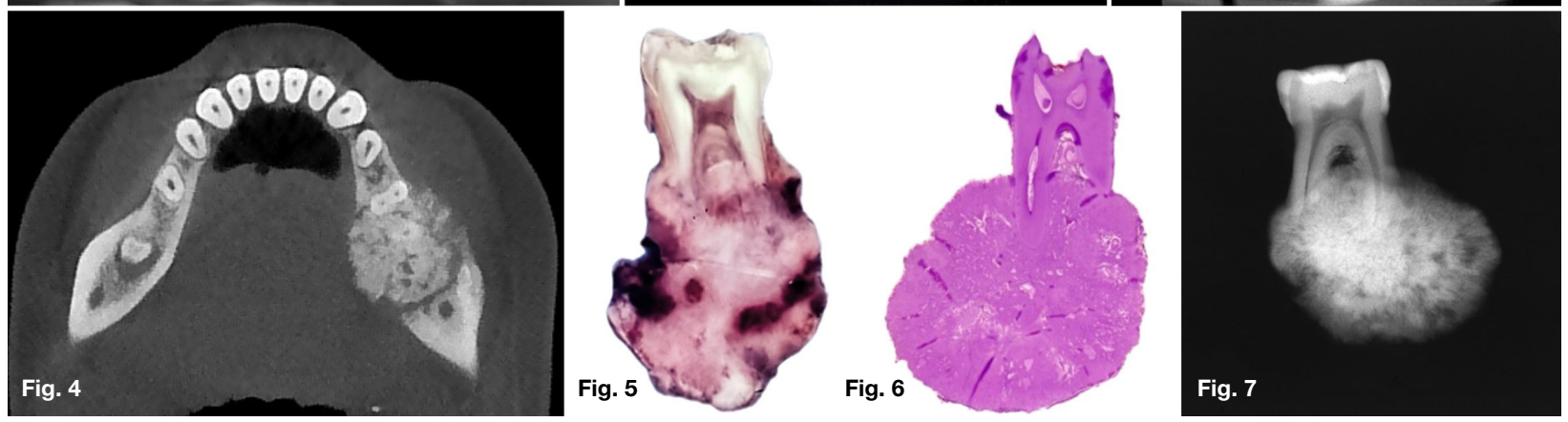

\section{INTERPRETATION}

CBCT imaging and analysis was performed. Observe unilateral expansion by the distending soft tissue outline illustrated through 3D rendering (Figure 2). Sagittal oblique (Figure 3) and axial (Figure 4) slices depict a round heterogenous predominantly high-density lesion with an encompassing thin uniform less dense peripheral band. Irregular root resorption, displacement of the inferior alveolar nerve canal, buccal-lingual cortical expansion, thinning, and interruption was apparent. Irregular thickening at the inferior border and surrounding osteosclerosis were noted. A macroscopic view (Figure 5), photomicrograph (Figure 6), and conventional radiograph (Figure 7) of vertically sectioned surgical specimens of similar lesions. Note the intimate relationship with the tooth roots.

The cementoblastoma is a benign odontogenic tumour of ectomesenchymal origin. The $\mathrm{WHO}$ characterise it as

\section{Author affiliations:}

1. Jaco Walters: $B C h D$ PDD (MFR) PGD (OS) MSc (MFR). Department of Oral and Maxillofacial Radiology, Faculty of Dentistry, University of the Western Cape, Tygerberg Oral Health Centre, Francie Van Zijl Drive, Cape Town 7505, South Africa. ORCiD: 0000-0002-0593-6890

\section{Corresponding author: Jaco Walters}

Department of Oral and Maxillofacial Radiology, Faculty of Dentistry, University of the Western Cape, Tygerberg Oral Health Centre. South Africa.

E-mail: jawalters@uwc.ac.za

Tel: +27219373078 established sheets of cementum-like tissue containing large numbers of reversal lines being unmineralized at the periphery or active growth regions. Considered rare with a prevalence of $0.2 \%$ to $6.2 \%$ amongst odontogenic tumours. With an age range of 8 to 44 years and predilection for the second and third decades. Approximately 46\% are diagnosed before the age of 20 . Gender ratios are seemingly negligible.

The majority present in the posterior mandible involving the first permanent molar, with primary tooth involvement considered to be rare. Common presentation is a unilateral slow-growing firm expansile swelling. In contrast to benign odontogenic tumours' hallmarks, pain and occasional paraesthesia may present. Radiological features of an ovoid radiopaque mass fused with a tooth root surrounded by a thin well-demarcated radiolucent zone are characteristic. Root resorption, periodontal ligament space and pulp chamber obliteration are common findings. Appearance may vary depending on the degree of mineralization. Early lesions are more radiolucent with increasing density as they mature. Due to persistent growth and involvement of adjacent structures removal is recommended by complete enucleation. A low rate of recurrence is reported.

\section{References}

1. Langlais, R. P., Langland, O. E., \& Nortjé, C. J. (1995). Diagnostic Imaging of the Jaws. Williams \& Wilkins.

2. Reichart, P., \& Philipsen, H. P. (2004). Odontogenic Tumors and Allied Lesions. Quintessence. 